\title{
The interaction between prognostic and pharmacodynamic biomarkers
}

\author{
L Bouranis ${ }^{1}$, M Sperrin ${ }^{1}$, A Greystoke ${ }^{2}$, C Dive ${ }^{2}$ and A G Renehan ${ }^{\star, 2,3}$ \\ ${ }^{1}$ Department of Mathematics and Statistics, Fylde College, Lancaster University, Lancaster, UK; ${ }^{2}$ Clinical and Experimental \\ Pharmacology (CEP) Group, Paterson Institute for Cancer Research, University of Manchester, Manchester, UK and \\ ${ }^{3}$ Faculty Institute of Cancer Sciences, University of Manchester, Manchester, UK
}

Background: Interactions between prognostic and pharmacodynamic (PD) biomarkers have received little attention.

Methods: Prognostic and PD utilities were assessed with linear mixed-effects models using published data on repeated measurements of circulating caspase-cleaved (ctCK18) and total (tCK18) cytokeratin 18, in 57 patients with metastatic colorectal cancer undergoing chemotherapy.

Results: The model for tCK18 (but not cCK18) separated the prognostic/PD interaction from the pure prognostic effect, illustrating the principle of dual prognostic and PD characteristics for a given biomarker.

Conclusion: These models provide the framework for the analysis and interpretation of longitudinal data to detect prognostic/PD biomarker interactions.

With the introduction of mechanism-based or 'targeted' therapies in oncology, there is a need to develop biomarkers to prioritise new agent development (predictive and prognostic), optimise dosing schedules (pharmacodynamics, PD), and rationalise combination selection (Tan et al, 2009; Cummings et al, 2010). Prognostic biomarkers provide evidence about a patient's disease outcome independent of treatment; predictive biomarkers assess the probability of benefit or toxicity from a specific intervention; and PD biomarkers provides evidence that there is a direct pharmacological effect of a drug (Dancey et al, 2010). The interaction between prognostic and predictive biomarkers is well established (Peterson and George, 1993; Mandrekar and Sargent, 2009; Buyse et al, 2010), but possible interactions between prognostic and $\mathrm{PD}$ biomarkers have received little attention. This is clinically important for a given biomarker, as the magnitude and/or direction of changes in PD characteristics may differ depending on its baseline prognostic level.

We previously published on repeated measurements of circulating caspase-cleaved and total cytokeratin 18 (ctCK18; tCK18), as biomarkers of epithelial cell death, in patients with metastatic colorectal cancer (mCRC) undergoing conventional chemotherapy, and provided evidence that serum concentrations of tCK18 are both prognostic and PD (Greystoke et al, 2012). However, in that study, the analysis was limited to simple regression modelling. Here, we assess prognostic and PD utilities within a linear mixed-effects framework, which takes account of intra-individual correlation between repeated biomarker measurements and allows for changes in the variance of the data with time.

\section{MATERIALS AND METHODS}

Fifty-seven patients with mCRC underwent repeated sampling during conventional chemotherapy, in a single cancer institute, as detailed elsewhere (Greystoke et al, 2012). The timings of repeated sampling varied depending on clinical practice, but were typically collected on days $1,3,8,15,21,28,35,42,49$, and 56, and thereafter determined by clinical indication.

We measured tCK18 and cCK18, respectively, using validated M65 and M30 ELISAs (Greystoke et al, 2008). Response to therapy was assessed by CT scan every 2 months during therapy. 
Statistical analysis. Prognostic characteristics were categorised as progressive disease or clinical benefit (stable or partial response combined) based on RECIST 1.0 (Therasse et al, 2000). PD characteristics were modelled from the repeated biomarker measurements and truncated at 120 days to avoid biases of unbalanced censoring. We fitted linear mixed-effects models (Weiss, 2005), separately for cCK18 and tCK18, after log transformation. Models incorporated a random intercept for the population and random slopes for the linear and quadratic time effects. We included type of chemotherapy treatment (first line $v s$ others) as an explanatory variable and tested the significance of the time-by-response interaction, both as fixed effects.

The models were estimated using restricted maximum likelihood. Forward selection was used to determine which fixed effects to include; Akaike Information Criterion was used to decide the optimum model. The assumptions of linearity, normality of residuals, and random effects, homoscedasticity and independence of within-subject errors were checked using diagnostic plots (Fitzmaurice et al, 2008). All analyses were performed using $\mathrm{R}$ statistical software (The $\mathrm{R}$ Project for Statistical Computing; http://www.r-project.org/). A $P$-value of 0.05 was taken as statistically significant.

\section{RESULTS}

Compared with patients experiencing clinical benefit, those with disease progression had higher median baseline cCK18 (383 vs $225 \mathrm{Ul}^{-1} ; P=0.019$ ) and tCK18 (999 vs $\left.389 \mathrm{Ul}^{-1} ; P=0.004\right)$ levels. In 25 patients with progressive disease, there was an initial decrease in both biomarker levels during cycle 1 followed by a progressive upward trend thereafter (Figure 1). In 32 patients with clinical benefit, an initial decrease in biomarker was observed in tCK18 only, with no subsequent change.

Longitudinal chemotherapy-related effects. For cCK18, fixed effects and random intercept and random slope models were tested, including time-by-response interactions, either in linear or quadratic forms. All two-way interactions were tried, but none found to be significant leading to a model described by the equation 1 in Supplementary Material 1. The optimum model succeeded in expressing the prognostic characteristics of cCK18, as the two lines have different starting points in the $y$ axis - the intercepts for clinical benefit and progressive disease were 214 and $355 \mathrm{Ul}^{-1}$, respectively, similar to the observed mean values (Figure 2). This model did not incorporate a time-by-response interaction, suggesting that there is not a prognostic/PD interaction, and making it irrelevant to separate out the prognostic/PD interaction from the pure prognostic effect.

For tCK18, the optimal model (equation 2, Supplementary Material 2) succeeded in capturing the prognostic characteristics, with the two curves having different starting points on the $y$ axis the intercepts for clinical benefit and progressive disease, respectively, were 464 and $896 \mathrm{Ul}^{-1}$ (Figure 3). The model incorporates a significant quadratic time-by-response interaction. The progressive disease group has a steeper curve for mean tCK18 concentration, increasing in time (approximately 12\% every 10 days). On the other hand, the curve for the clinical benefit group tends to be relatively flat. This model had the ability to capture the PD utility of tCK18, whereas separating out the prognostic/PD interaction from the pure prognostic effect.

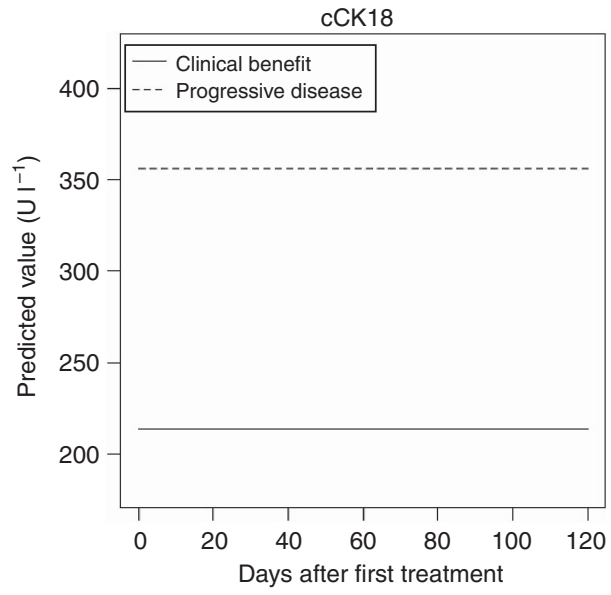

\begin{tabular}{lccc}
\hline Fixed effects & Coef. & $95 \% \mathrm{Cl}$ & $P$-value \\
\hline Intercept & 5.365 & $(5.176,5.554)$ & $<0.001$ \\
$\begin{array}{l}\text { Response: progressive } \\
\text { disease }\end{array}$ & 0.507 & $(0.209,0.806)$ & 0.001 \\
\hline
\end{tabular}

Figure 2. Predicted values ( $y$ axis) from the multivariate linear mixed-effects model of the log-transformed caspase-cleaved CK18 (cCK18), measured in the original scale. Each line corresponds to a different type of response to treatment. The table shows the fixed effects components for the optimal fitting model - the complete model is reported in the Supplementary Material. The intercepts for clinical benefit was $214 \mathrm{UI}^{-1}$ (anti- $\left.\log _{\text {natural }}[5.365]\right)$; the intercept for progressive disease was $355 \mathrm{UI}^{-1}[214+(214 \times$ (anti-lognatural [0.507] -1))].
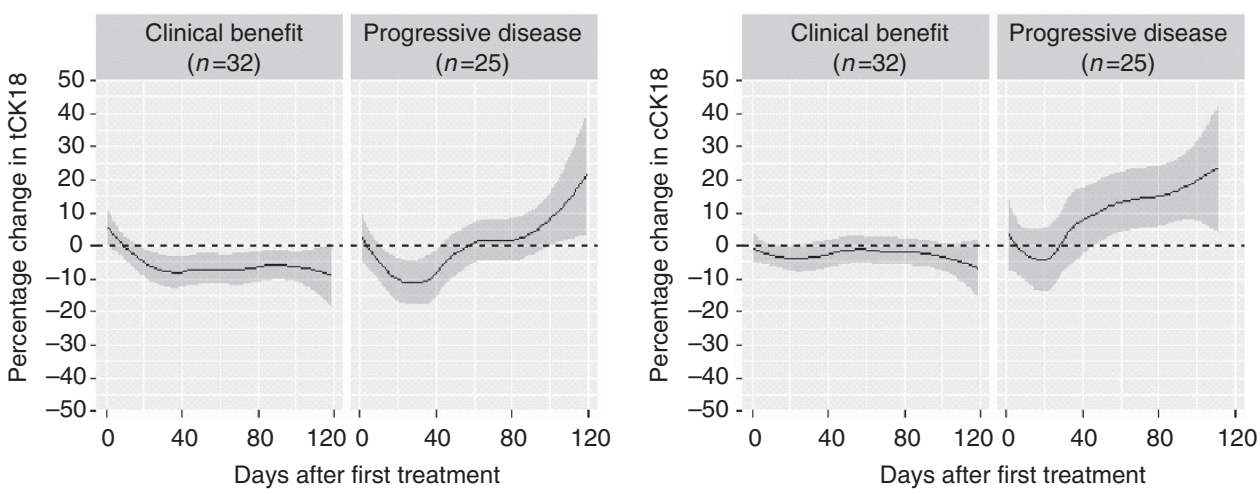

Figure 1. PD monitoring of tCK18 and cCK18 in 57 patients with metastatic CRC undergoing conventional chemotherapy, categorised by treatment response. The $y$ axis is percentage change in biomarker, taken against value at day 1 . Curves were generated using Lowess smoother. Shaded area represents $95 \% \mathrm{Cl}$. 


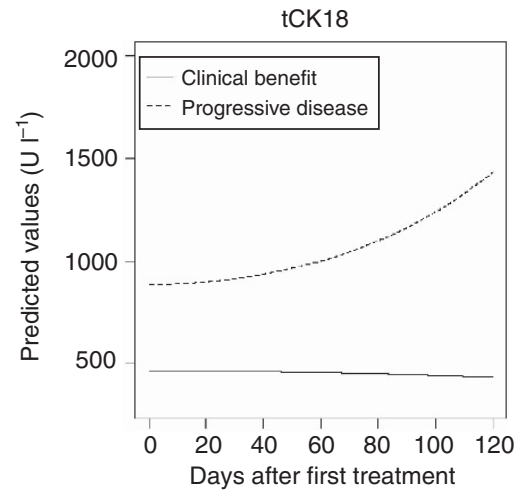

\begin{tabular}{lccc}
\hline Fixed effects & Coef. & $95 \% \mathrm{Cl}$ & $P$-value \\
\hline Intercept & 6.139 & $(5.901,6.378)$ & $<0.001$ \\
$t^{2}{ }_{\mathrm{ij}}$ & -0.077 & $(-0.238,0.084)$ & 0.346 \\
Response: progressive disease $^{2}$ & 0.656 & $(0.288,1.024)$ & $<0.001$ \\
$t^{2}{ }_{\mathrm{ij}}$ x progressive disease & 0.511 & $(0.221,0.801)$ & $<0.001$
\end{tabular}

Figure 3. Predicted values ( $y$ axis) from the multivariate linear mixedeffects model of the log-transformed total CK18 (tCK18), measured in the original scale. Each curve corresponds to a different type of response to treatment. The table shows the fixed effects components for the optimal fitting model - the complete model is reported in the Supplementary Material. The intercepts for clinical benefit was $464 \mathrm{UI}^{-1}$ (anti-lognatural[6.139]; the intercept for progressive disease was $896 \mathrm{UI}^{-1}[464+(464 \times($ anti-lognatural $[0.656]-1))]$. The slope of the progressive disease group was approximated as $12 \%$ per 10 days (anti$\log _{\text {natural }}[-0.077+0.511]$ per unit $\left.\mathrm{t}^{2}\right) .{ }^{*} \mathrm{~A}$ number of models were built and tested against the least Akaike Information Criterion (AIC) value. For the model without the interaction term, $\mathrm{AIC}=261.5128$; for the model with the interaction term, $\mathrm{AIC}=257.7854$, and this model was preferred.

Diagnostic tests indicated no violations of the model assumptions (Supplementary Material 3-5).

\section{DISCUSSION}

PD biomarkers can provide evidence of drug action, help select an optimal drug dose and schedule, and inform on the design of combination therapy schedules (Sarker and Workman, 2007). Here, we have examined the utility of tCK18, a proof of concept PD biomarker of drug-induced epithelial cell death, in patients with metastatic CRC undertaking standard of care chemotherapy. We had previously determined that at baseline tCK18 was prognostic. Statistical modelling in this study indicated that patients with high baseline tCK18 concentrations (poor prognosis) exhibit a PD change in this biomarker, whereas patients with low baseline tCK18 (better prognosis) do not. The implication of these findings is that where the PD characteristics of a biomarker are being studied, there is a general principle that prognostic/PD interactions need to be explored.

Study strengths and limitations. Strengths of this study include the following. The linear mixed-effects models handle both timevarying and time-invariant covariates and provide a flexible, yet parsimonious model for the covariance, using likelihood-based methods to estimate the model parameters efficiently. Specifying a mixed model requires many steps (both a weakness and a strength), each of which requires an informed choice (Fitzmaurice et al, 2008). This study shows that the linear mixed models approach offers a feasible method to investigate interactions between the prognostic and PD characteristics of biomarkers, despite its complexity.

The study findings should be interpreted in light of some limitations. First, patient recruitment was within a standard care setting, such that treatment selection and follow-up protocols were not precisely controlled. This weakness was partly addressed by the inclusion of 'type of chemotherapy treatment' (first line $v s$ others) in the multivariate model. Second, treatment response was defined in retrospect. In clinical practice, researchers need to know the trajectories of the measurements early in the study, with the gold standard for biomarker qualification being prospective treatment protocol-driven clinical data.

Context of other studies. The utility of biomarkers as both prognostic and PD has been explored in a small number of previous studies. For example, circulating nDNA levels were elevated in patients with lymphoma (Deligezer et al, 2006) and lung cancer (Holdenrieder et al, 2008) compared with healthy controls and were demonstrated to have both prognostic and PD utilities. In a review of 25 studies of the utility of circulating tumour cells (CTCs) in early clinical trials, including breast, colorectal, and prostate cancer, Devriese et al (2011) reported that while the majority of studies comments on the prognostic significance of specific targets in CTCs, only five or $20 \%$ commented on the PD effects of these CTC targets.

None of the above studies addressed the issue of interactions between the prognostic and PD utility of biomarkers in cancer. The majority of studies implemented different survival analysis techniques, such as log-rank tests or multivariate Cox regression models for the assessment of the PD behaviour of different biomarkers, with the overall survival or progression-free survival being used as end points (Hahn et al, 2008; Kudo et al, 2011). As the distributions of biomarkers were positively skewed, some studies used non-parametric tests to satisfy the assumptions of unequal variance between sample groups (Greystoke et al, 2011).

Unanswered questions and future research. In our analysis, we did not account for patient censorship, but instead, minimised this potential bias by truncating the time period. In future studies, where censorship (invariably due to cancer-related death) is an issue, joint models for longitudinal and survival data would be an alternative approach (Ibrahim et al, 2010). These models would allow the repeated measurements to be extrapolated from the observed measurement times to the specific event time in a way that utilises the entire measurement history. Ultimately, the repeated measurement process would be adjusted for any loss of information arising from death or loss of individuals (Lang et al, 2012). However, this type of modelling increases the complexity.

The results presented in this paper show a need to recognise the interaction of the prognostic and PD characteristics of a biomarker, and to better understand the underlying mechanisms of this interaction in future studies.

\section{AUTHOR CONTRIBUTIONS}

LB, MS, and AGR contributed to study design, analyses, and data interpretation. $\mathrm{AG}$ and $\mathrm{CD}$ contributed to the pharmacological interpretation; LB and MS contributed to the statistical interpretation. All authors contributed and approved the final manuscript.

\section{CONFLICT OF INTEREST}

The authors declare no conflict of interest. 


\section{REFERENCES}

Buyse M, Sargent DJ, Grothey A, Matheson A, de Gramont A (2010) Biomarkers and surrogate end points-the challenge of statistical validation. Nat Rev Clin Oncol 7(6): 309-317.

Cummings J, Raynaud F, Jones L, Sugar R, Dive C (2010) Fit-for-purpose biomarker method validation for application in clinical trials of anticancer drugs. Br J Cancer 103(9): 1313-1317.

Dancey JE, Dobbin KK, Groshen S, Jessup JM, Hruszkewycz AH, Koehler M, Parchment R, Ratain MJ, Shankar LK, Stadler WM, True LD, Gravell A, Grever MR (2010) Guidelines for the development and incorporation of biomarker studies in early clinical trials of novel agents. Clin Cancer Res 16(6): 1745-1755.

Deligezer U, Erten N, Akisik EE, Dalay N (2006) Circulating fragmented nucleosomal DNA and caspase-3 mRNA in patients with lymphoma and myeloma. Exp Mol Pathol 80(1): 72-76.

Devriese LA, Voest EE, Beijnen JH, Schellens JH (2011) Circulating tumor cells as pharmacodynamic biomarker in early clinical oncological trials. Cancer Treat Rev 37(8): 579-589.

Fitzmaurice G, Davidian M, Verbeke G, Molenberghs G (2008) Longitudinal Data Analysis: A Handbook of Modern Statistical Methods. Taylor \& Francis: London.

Greystoke A, Cummings J, Ward T, Simpson K, Renehan A, Butt F, Moore D, Gietema J, Blackhall F, Ranson M, Hughes A, Dive C (2008) Optimisation of circulating biomarkers of cell death for routine clinical use. Ann Oncol 19(5): 990-995.

Greystoke A, Dean E, Saunders MP, Cummings J, Hughes A, Ranson M, Dive C, Renehan AG (2012) Multi-level evidence that circulating CK18 is a biomarker of tumour burden in colorectal cancer. Br J Cancer 107(9): 1518-1524.

Greystoke A, O'Connor JP, Linton K, Taylor MB, Cummings J, Ward T, Maders F, Hughes A, Ranson M, Illidge TM, Radford J, Dive C (2011) Assessment of circulating biomarkers for potential pharmacodynamic utility in patients with lymphoma. Br J Cancer 104(4): 719-725.

Hahn OM, Yang C, Medved M, Karczmar G, Kistner E, Karrison T, Manchen E, Mitchell M, Ratain MJ, Stadler WM (2008) Dynamic contrast-enhanced magnetic resonance imaging pharmacodynamic biomarker study of sorafenib in metastatic renal carcinoma. J Clin Oncol 26(28): 4572-4578.

Holdenrieder S, Nagel D, Schalhorn A, Heinemann V, Wilkowski R, von Pawel J, Raith H, Feldmann K, Kremer AE, Muller S, Geiger S, Hamann GF, Seidel D, Stieber P (2008) Clinical relevance of circulating nucleosomes in cancer. Ann N Y Acad Sci 1137: 180-189.

Ibrahim JG, Chu H, Chen LM (2010) Basic concepts and methods for joint models of longitudinal and survival data. J Clin Oncol 28(16): 2796-2801.

Kudo K, Arao T, Tanaka K, Nagai T, Furuta K, Sakai K, Kaneda H, Matsumoto K, Tamura D, Aomatsu K, De Velasco MA, Fujita Y, Saijo N, Kudo M, Nishio K (2011) Antitumor activity of BIBF 1120, a triple angiokinase inhibitor, and use of VEGFR2 + pTyr + peripheral blood leukocytes as a pharmacodynamic biomarker in vivo. Clin Cancer Res 17(6): 1373-1381.

Lang W, Wei L, Grace Y, Yangxin H (2012) Analysis of longitudinal and survival data: joint modeling, inference methods, and issues. J Probab Stat 2012. http://dx.doi.org/10.1155/2012/640153.

Mandrekar SJ, Sargent DJ (2009) Clinical trial designs for predictive biomarker validation: theoretical considerations and practical challenges. J Clin Oncol 27(24): 4027-4034.

Peterson B, George SL (1993) Sample size requirements and length of study for testing interaction in a $2 \mathrm{x} \mathrm{k}$ factorial design when time-to-failure is the outcome [corrected]. Control Clin Trials 14(6): 511-522.

Sarker D, Workman P (2007) Pharmacodynamic biomarkers for molecular cancer therapeutics. Adv Cancer Res 96: 213-268.

Tan DS, Thomas GV, Garrett MD, Banerji U, de Bono JS, Kaye SB, Workman P (2009) Biomarker-driven early clinical trials in oncology: a paradigm shift in drug development. Cancer J 15(5): 406-420.

Therasse P, Arbuck SG, Eisenhauer EA, Wanders J, Kaplan RS, Rubinstein L, Verweij J, Van Glabbeke M, van Oosterom AT, Christian MC, Gwyther SG (2000) New guidelines to evaluate the response to treatment in solid tumors. European Organization for Research and Treatment of Cancer, National Cancer Institute of the United States, National Cancer Institute of Canada. J Natl Cancer Inst 92(3): 205-216.

Weiss RE (2005) Modeling Longitudinal Data.: Springer Texts in Statistics. Springer World Health: New York.

Supplementary Information accompanies this paper on British Journal of Cancer website (http://www.nature.com/bjc) 